\title{
Deciduous Dentitions of Eocene Cebochoerid Artiodactyls and Cetartiodactyl Relationships
}

\author{
Jessica M. Theodor $\cdot$ Scott E. Foss
}

Published online: 8 June 2006

(C) Springer Science+Business Media, Inc. 2006

\section{Journal of Mammalian Evolution (2005) 12(1/2):161-181}

In the article by Theodor and Foss, Figs. 3 and 4 appeared with incorrect legends. The figures are presented here, with the correct legends.

The online version of the original article can be found at http://dx.doi.org/10.1007/s10914-005-5706-6

J. M. Theodor $(\square)$

Department of Geology, Illinois State Museum, Springfield, Illinois 62703, USA

e-mail: jtheodor@museum.state.il.us

S. E. Foss

John Day Fossil Beds National Monument, Kimberly, Oregon 97848, USA 
a
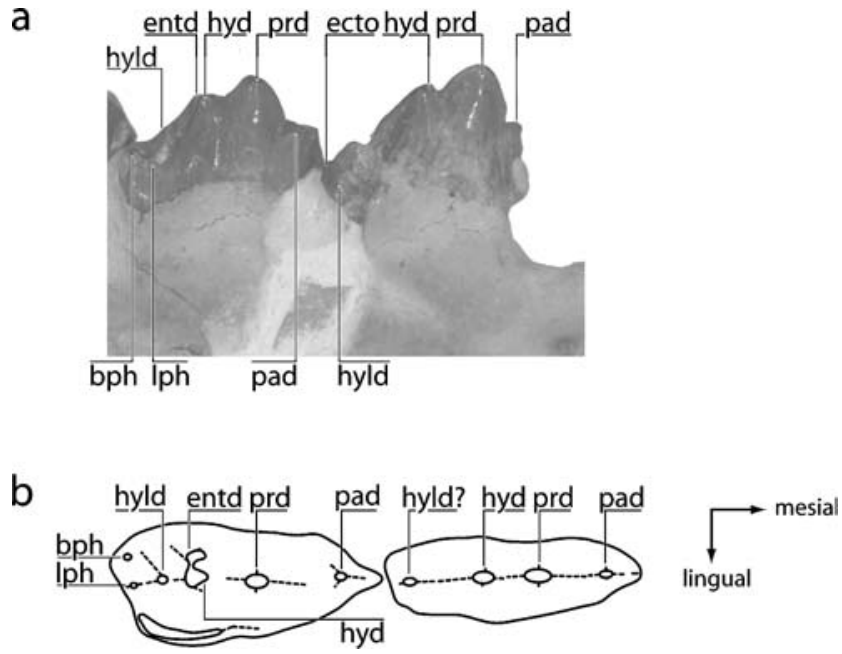

Fig. 3 Cusp terminology for deciduous lower premolars of cebochoerids. (a) lingual view of dp23, MNHN.EBA327, (b) occlusal view of dp2-3. bph-buccal posthypoconulid; ecto-ectostylid; entdentoconid; hyd-hypoconid; hyld-hypoconulid; lph—lingual posthypoconulid; pad-paraconid; prdprotoconid

a

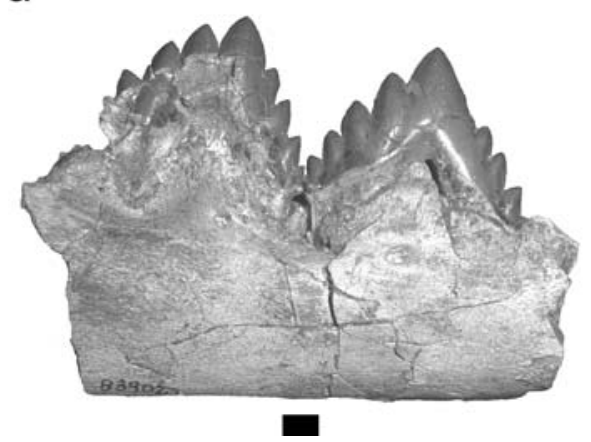

b

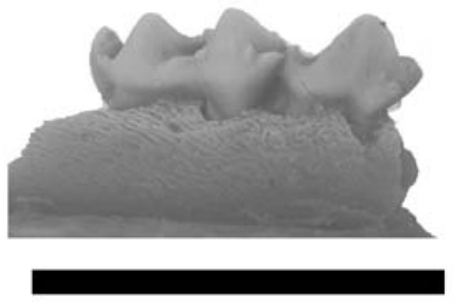

Fig. 4 Deciduous teeth of (a) Dorudon atrox, UM 83902, buccal view dp3-4, photograph courtesy of Mark Uhen. (b) Antiacodon pygmaeus, CMNH 53969, buccal view dp3-4. Scale bars denote $1 \mathrm{~cm}$ 